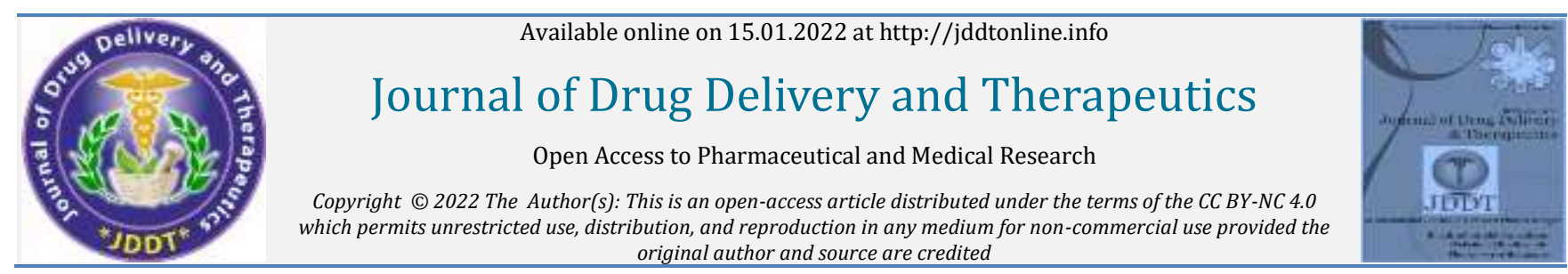
original author and source are credited

Research Article

\title{
Oxaliplatin Induced Severe Hypoxemia, Chills and Hypersensitivity
} Reaction

\author{
Syeda Zaineb Humaira Hussaini ${ }^{*}$, Faiza Aftab ${ }^{2}$, Nisma Siddiqi ${ }^{3}$, Syed Ishtiaq Ahmed ${ }^{3}$, Syed Abdul \\ Rehman $^{3}$ \\ ${ }^{1}$ Assistant Professor, Department of Pharmacy Practice, Bhaskar Pharmacy College, Hyderabad, India \\ ${ }^{2}$ MBBS, Former Medical Officer, Department of Medical Oncology, Basavatarakam Indo-American Cancer Hospital and Research Institute, \\ Hyderabad, India \\ ${ }^{3}$ Pharm-D (Doctor of Pharmacy), Bhaskar Pharmacy College, Hyderabad, Telangana, India
}

\section{Article Info:}

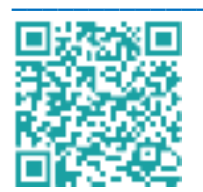

\section{Article History}

Received 10 November 2021 Reviewed 26 December 2021 Accepted 04 January 2022

Published 15 January 2022

\section{Cite this article as:}

Hussaini SZH, Aftab F, Siddiqi N, Ahmed SI, Rehman SA, Oxaliplatin Induced Severe Hypoxemia, Chills and Hypersensitivity Reaction, Journal of Drug Delivery and Therapeutics. 2022; 12(1):39-41

DOI: http://dx.doi.org/10.22270/jddt.v12i1.5278

\section{*Address for Correspondence:}

Dr. Syeda Zaineb Humaira Hussaini, Assistant Professor, Department of Pharmacy Practice, Bhaskar Pharmacy College, Hyderabad, India

\section{Abstract}

Hypersensitivity reactions to platinum containing compounds are well described and potentially life threatening. The hypersensitivity reactions can occur either during, or shortly after the infusion of drug. The incidence increases with increase in the number of chemotherapy cycles. Hypersensitivity reactions can vary from mild reactions like rashes to severe reactions, which include laryngospasm, tachycardia, hypotension or hypertension. The combination of 5-flurouracil, leucovarin and oxaliplatin has been used in several studies to increase survival rates and reduce the risk of disease progression in patients with metastatic colorectal cancer (CRC) and stage III colon cancer. We report a case of patient treated with oxaliplatin who developed Severe Grade-III Toxicity including Hypoxemia, Chills and hypersensitivity reaction like rashes all over the body with itching sensation about few hrs after the oxaliplatin infusion. Patient tolerated 7 cycles of FLOT chemotherapy well. During cycle 8 chemotherapy after $50 \%$ infusion of oxaliplatin patient developed severe reaction to oxaliplatin such as breathlessness, shivering, hypersensitivity reaction like rashes all over the body and itching sensation. The drug infusion was stopped immediately, vitals were checked which shows BP: $170 / 110 \mathrm{mmhg}$, Temperature $100^{\circ} \mathrm{F}$, Spo 2 85\% RA. It was confirmed as grade 3 toxicity of oxaliplatin. The adverse drug reaction assessment was done using Naranjo's causality assessment scale which showed 'definite' type of adverse drug reaction with oxaliplatin. Prompt recognition of this event and symptomatic treatment with supplemental oxygen and corticosteroids and prolonging the infusion time of oxaliplatin can lead to better patient compliance and lesser hypersensitivity reactions to these regimens.

Keywords: Oxaliplatin, Colorectal cancer (CRC), Dyspnoea, Hypersensitivity reactions, Chemotherapy.

\section{INTRODUCTION:}

Oxaliplatin, a third-generation platinum compound, one of the mainstay drugs in the treatment of many gastrointestinal cancers, can give rise to hypersensitivity reactions, sometimes with fatal outcomes. It is usually combined with 5-flurouracil (5-FU), capecitabine, irinotecan, or cyclophosphamide. It is a non-cell cycle specific alkylating agent that causes abnormal crosslinking or cutting of DNA strands and eventually leading to cell death.

Hypersensitivity reactions to platinum containing compounds are well described and potentially life threatening. These reactions were thought to be less frequent with oxaliplatin compared with cisplatin or carboplatin, but as a result of expanded use of oxaliplatin in various malignancies, an increased number of hypersensitivity reactions have been reported with an incidence of $10-12 \%$ including a rate of $>1 \%$ for severe reactions 1 .
The hypersensitivity reactions can occur either during, or shortly after the infusion of drug. The incidence increases with increase in the number of chemotherapy cycles. Hypersensitivity reactions can vary from mild reactions like rashes to severe reactions, which include laryngospasm, tachycardia, hypotension or hypertension.

Oxaliplatin is less nephrotoxic than cisplatin and less myelotoxic than carboplatin. The most characteristic and dose limiting toxicity of oxaliplatin is sensory neuropathy, transient acute cold related dysesthesias, sometimes pain associated although it is generally reversible. Hypersensitivity reactions to oxaliplatin have been described as anaphylaxis. This reaction is clinically characterised by laryngospasm and wheezing and immunologically linked to the release of histamine and other vasoactive substances.

Oxaliplatin has been widely used in patients with gastrointestinal malignancies including colorectal cancer (CRC). 
The combination of 5-flurouracil, leucovarin and oxaliplatin (FOLFOX) has been used in several studies to increase survival rates and reduce the risk of disease progression in patients with metastatic CRC and stage III colon cancer 2,3

Hypersensitivity is a possible adverse effect of exposure to platinum compounds and the incidence increases with multiple cycles of therapy ${ }^{4-6}$. The hypersensitivity reaction associated with oxaliplatin typically consists of rigors, fever, rashes, tachycardia, and dyspnoea. The incidence in patients with CRC was reported as high as $15 \%$ and mainly occurred shortly after infusion in patients who had prior exposure to oxaliplatin 7,8

The mild hypersensitivity reaction (grade 1 and 2) usually responds to discontinuation of oxaliplatin and supportive treatment with antihistamine agents and steroid. Frequently, patients with mild hypersensitivity reaction can be retreated with oxaliplatin by adding appropriate premeditations such as antihistamine agents and steroids and increasing infusion time with more diluted concentration 8,9

Severe and potentially fatal hypersensitivity reaction with symptomatic bronchospasm, angioedema, hypotension and anaphylaxis occurred on $2 \%$ patients receiving oxaliplatin treatment 10,11 . Although the manufacturer recommends not to re-treat with oxaliplatin after the incidence of severe hypersensitivity reaction, a desensitization protocol has been successfully implemented in patients with grade 3 hypersensitivity reaction 12 .

Here, we report a case of patient treated with oxaliplatin who developed Severe Grade-III Toxicity including Hypoxemia, Chills and hypersensitivity reaction like rashes all over the body with itching sensation about few hrs after the oxaliplatin infusion during $8^{\text {th }}$ cycle of FLOT Chemotherapy.

\section{CASE REPORT:}

A 54-year-old male patient was presented with epigastric pain and early satiety in the past 6 months. He was diagnosed as gastric carcinoma stage III PT3N3bM0. Endoscopy revealed presence of ulcerative growth in the stomach. Biopsy from the lesion showed presence of moderately differentiated adenocarcinoma, diffuse type, poorly differentiated. Tumour located in body, pylorus and antrum. Tumour measures $7 \mathrm{~cm}$ in dimensions, tumour invades subserosal fat and reaches up to serosal margin. Lymphovascular invasion is identified. Proximal and distal are margins are free of tumour. 29 out of 48 lymphnodes show metastatic tumour deposit, size of large deposit measures $1 \mathrm{~cm}$ in greatest dimensions with extranodal extension.

He underwent laproscopy of distal gastrectomy + D2 lymphadenopathy + roux-en-y-gastrojejunostomy + jejunojenostomy + feeding jejunostomy + splenectomy on $10 / 3 / 2020$ laboratory tests for chest X-ray PA view, CBP, blood for culture and sensitivity, HIV 1 and 2, USG whole abdomen were within normal limits. Multislice spiral CT whole abdomen revealed focal circumferential wall thickening in distal part of body of stomach and gastric antrum showing significant enhancement and multiple perilesional lymphnodes and fat stranding S/O malignant gastric wall thickening. UGI scopy revealed proliferative mass in lesser curvature, indicating stomach cancer. Histopathology report of surgical specimen revealed adenocarcinoma poorly differentiated PT3N3bM0, lymphovascular invasion present, perinodal extension present. He was planned for adjuvant chemotherapy with
FLOT regimen. Completed cycle 7 chemotherapy and was asked to review after $21^{\text {st }}$ day for cycle 8 FLOT regimen.

Chemotherapeutic drugs for FLOT regimen include Inj. fluorouracil 300mg in Baxter pump approximately for 48 hrs, Inj. Docetaxel $60 \mathrm{mg}$ in $500 \mathrm{ml}$ normal saline $\sim 21 / 2$ hours, Inj. leucovorin $270 \mathrm{mg}$ in $100 \mathrm{ml}$ normal saline $\sim 1$ hour, and Inj. oxaliplatin $110 \mathrm{mg}$ in $500 \mathrm{ml} 5 \%$ dextrose $\sim 2$ $1 / 2$ hours.

Patient tolerated 7 cycles of chemotherapy well. During cycle 8 chemotherapy after $50 \%$ infusion of oxaliplatin patient developed severe reaction to oxaliplatin such as breathlessness, shivering, hypersensitivity reaction like rashes all over the body and itching sensation. The drug infusion was stopped immediately, vitals were checked which shows BP: $170 / 110 \mathrm{mmhg}$, Temperature $100^{\circ} \mathrm{F}$, Spo 2 $85 \%$ RA. It was confirmed as grade 3 toxicity of oxaliplatin. He was prescribed with Inj. Hydrocort stat, Inj. Avil 1 Amp stat, Inj. Paracetamol 1gm IV stat were given. Continuous vital monitoring was done. When symptoms were improved and vitals were stable (nearly after 30 to $45 \mathrm{~min}$ ). infusion was re-started with dose reduction rate. Within $5 \mathrm{~min}$ of infusion patient again developed itching and rashes on hands. Infusion was stopped and discarded. Patient was kept 1 day for observation and then got discharged in stable condition.

The adverse drug reaction assessment was done using Naranjo's causality assessment scale which showed 'definite' type of adverse drug reaction with oxaliplatin. The patient was asked to review after 1 month for re-evaluation with CBP, serum creatinine and serum bilirubin.

\section{DISCUSSION:}

Hypersensitivity to platinum derivatives was first reported in 200413. A second report of delayed oxaliplatin induced laryngeal spasm was reported in $2009{ }^{14}$. Another report showing ADR developed 24 hours after the 1st and 2nd dose infusion of oxaliplatin 15

Oxaliplatin a coordination compound of DACH carrier group and an oxalate leaving group was active in cisplatin resistant tumour models. Like cisplatin oxaliplatin preferentially forms adducts at thN7 position of guanine and to lesser extent adenine however there is evidence that the 3 dimensional structure of DNA adducts and biologic response they elicit are different from those of cisplatin 16

In the immediate onset cases, the patient showed various symptoms. In previous reports; approximately $40 \%$ of allergic patients showed rash or cutaneous reactions 17,18 . In our case the patient had breathlessness, shivering, hypersensitivity reaction like rashes all over the body and itching sensation, which was treated with Inj. Hydrocort stat, and Inj. Avil 1 ampule stat. Hypertension and dyspnea were severe complaints, and in our case, patient had a reaction of grade 3 Toxicity.

In a case study conducted by Siu et al five patients with grade 3 severity required oxygen. Siu et al (18) found rates of $14.8 \%$ for dyspnea and $7.4 \%$ for hypotension in patients with hypersensitivity to oxaliplatin. These results suggest that respiratory problems due to oxaliplatin allergy may be more severe than initially apparent, and a saturation monitor should always be used in cases of immediate onset allergy.

According to a MEDLINE search for recent and past studies, case reports, metanalysis and review pertaining to oxaliplatin related hypersensitivity reactions are performed, the mechanism for oxaliplatin induced hypersensitivity 
reaction is associated with immunoglobulins Ig-E mediated hypersensitivity ${ }^{19}$.

The hypersensitivity reactions can be minimised by increasing the infusion time from two hours to six hours. Administration of intravenous calcium gluconate and magnesium sulphate, $1 \mathrm{~g}$ each, just before the oxaliplatin infusion is reported to decrease the incidence of acute neurotoxicity as well as laryngospasm especially pseudo laryngospasm. 20

Re-introduction of therapy was tried to this patient and he showed relapse of allergy. Therefore, re-introduction of therapy requires premedication with steroids and histamine receptor antagonists, and patients should be monitored closely for relapse of allergy. The adverse drug reaction was assessed by using Naranjo's Causality Assessment Scale which showed definite type of reaction with oxaliplatin.

Our study aims to contribute awareness about the adverse drug reaction related to oxaliplatin. As oxaliplatin is approved for the treatment of advanced colorectal cancer and enhances cure rate in adjuvant setting. The therapeutic role of oxaliplatin has been found to extend to pancreatic gastric and oesophageal cancer. Close monitoring is required while infusing oxaliplatin for prompt identification and management of oxaliplatin induced hypersensitivity reaction, which may occur after many hours of oxaliplatin infusion. Overall, our case suggests that oxaliplatin allergy is an important concern and that methods for suppression of allergic response is required.

\section{CONCLUSION:}

This case report suggests that oxaliplatin has a propensity to cause severe hypersensitivity reactions manifesting as Hypoxemia and chills with subsequent doses of infusion and not with a single dose. As use of this agent becomes more widespread, increased vigilance for this potentially serious complication should be high, Previous exposure to oxaliplatin is a risk factor for earlier Hypersensitivity Reaction onset and more severe and frequent Hypersensitivity Reaction episodes, even if prior therapy was well tolerated. Prompt recognition of this event and symptomatic treatment with supplemental oxygen and corticosteroids and prolonging the infusion time of oxaliplatin can lead to better patient compliance and lesser hypersensitivity reactions to these regimens.

\section{REFERENCES:}

1. Lenz H-J. Management and preparedness for infusion and Hypersensitivity reactions. Oncologist 2007; 12:601-9 https://doi.org/10.1634/theoncologist.12-5-601

2. Goldberg RM, Sargent DJ, Morton RF, Fuchs CS, Ramanathan RK,Williamson SK, Findlay BP, Pitot HC, Alberts SR: A randomized controlled Trial of fluorouracil plus leucovorin, irinotecan, and oxaliplatin Combinations in patients with previously untreated metastatic colorectal Cancer. J Clin Oncol 2004; 22(1):23-30. https://doi.org/10.1200/JC0.2004.09.046

3. Andre T, Boni C, Mounedji-Boudiaf L, Navarro M, Tabernero J, Hickish T, Topham C, Zaninelli M, Clingan P, Bridgewater J, et al: Oxaliplatin, Fluorouracil, and leucovorin as adjuvant treatment for colon cancer.N Engl J Med 2004; 350(23):2343-2351. https://doi.org/10.1056/NEJMoa032709

4. Markman M, Kennedy A, Webster K, et al. Clinical features Of hypersensitivity reactions to carboplatin. J Clin Oncol.1999; 17:1141-1145. https://doi.org/10.1200/JC0.1999.17.4.1141
5. Shlebak AA, Clark PI, Green JA. Hypersensitivity and crossreactivity to cisplatin and analogues. Cancer Chemother Pharmacol. 1995; 35:349-351. https://doi.org/10.1007/BF00689458

6. Polyzos A, Tsavaris N, Kosmas C, et al. Hypersensitivity Reactions to carboplatin administration are common but Not always severe: a 10-year experience. Oncology. 2001; 61:129-133. https://doi.org/10.1159/000055363

7. Brandi G, Pantaleo MA, Galli C, Falcone A, Antonuzzo A, Mordenti P, Di Marco MC, Biasco G: Hypersensitivity reactions related to oxaliplatin (OHP). Br J Cancer 2003; 89(3):477-481 https://doi.org/10.1038/sj.bjc.6601155

8. Siu SWK, Chan RTT, Au GKH: Hypersensitivity reactions to oxaliplatin:Experience in a single institute. Ann Oncol 2006, 17(2):259-261. https://doi.org/10.1093/annonc/mdj042

9. Gowda A, Goel R, Berdzik J, Leichman CG, Javle M: Hypersensitivity Reactions to oxaliplatin: incidence and management. Oncology (WillistonPark) 2004

18(13):1671-1675, discussion 1676, 1680, 1683-1674.

10. ELOXATIN Prescribing information. [http://products.sanofiaventis.us/Eloxatin/eloxatin.html].

11. Lee MY, Yang MH, Liu JH, Yen CC, Lin PC, Teng HW, Wang WS, Chiou TJ,Chen PM: Severe anaphylactic reactions in patients receiving oxaliplatin therapy: a rare but potentially fatal complication. Support Care Cancer 2007; 15(1):89-93. https://doi.org/10.1007/s00520-006-0107-9

12. Gammon D, Bhargava P, McCormick MJ: Hypersensitivity reactions to Oxaliplatin and the application of a desensitization protocol. Oncologist 2004; 9(5):546-549. https://doi.org/10.1634/theoncologist.9-5-546

13. Gowda A, Goel R, Berdzik J et al, Hypersensitivity Reactions to oxaliplatin: incidence and management. Oncology 2004; 18:1671-1675

14. Simona Potenza, Guglielmo Nasti et al (2009) severe respiratory symptoms to oxaliplatin infusion : A case report of delayed hypersensitivity reaction. Invest new drugs 2010; 28:185-186 DOI 10.1007/S 10637-009-9250-8 https://doi.org/10.1007/s10637-009-9250-8

15. de Vries RS, Mattijssen EJ, van Sorge AA, Serious delayed hypersensitivity reaction to oxaliplatin. Ann Oncol 2006; 17:1723-1724. doi:10.1093/annonc/mdl116 https://doi.org/10.1093/annonc/mdl116

16. Johnstone TC, Suntharalingam K, Lippard SJ. The next generation of platinum drugs: targeted $\mathrm{Pt}(\mathrm{II})$ agents, nanoparticle delivery, and Pt(IV) prodrugs. Chem Rev 2016; 116(5):3436-3486. https://doi.org/10.1021/acs.chemrev.5b00597

17. Maindrault-Goebel F, André T, Tournigand C, Louvet C, PerezStaub N, Zeghib N, et al. Allergic-type reactions to oxaliplatin: retrospective analysis of 42 patients, Eur J Cancer, 2005; 41:2262-7 https://doi.org/10.1016/j.ejca.2005.06.021

18. Siu SW, Chan RT, Au GK. Hypersensitivity reactions to oxaliplatin: experience in a single institute, Ann Oncol, 2006; 17:259-61 https://doi.org/10.1093/annonc/mdj042

19. Lenz HJ. Management and preparedness for infusion and hypersensitivity reactions. Oncologist. 2007; 12(5):601-9. https://doi.org/10.1634/theoncologist.12-5-601

20.Gamelin L, Boisdron-celle M, Delva R, Ifrah N, Morel A, Gamelin E. Prevention of Oxaliplatin Related Neurotoxicity by Calcium and Magnesium Infusions: A Retrospective Study of 161 Patients Receiving Oxaliplatin Combined with 5-Fluorouracil and Leucovorin for Advanced Colorectal Cancer. Clin Cancer Res. 2004; 10(12):4055-61. https://doi.org/10.1158/10780432.CCR-03-0666 